\title{
Analysis and design of computer aided 3D physical training system
}

\author{
SUN Baocong ${ }^{1, \text { a }}$ \\ ${ }^{1}$ School of physical education, Shandong University of Finance \& Economics, Jinan City \\ 250014, china \\ a sunbcty@126.com
}

Keywords: Computer-Assisted Three-Dimensional; Sports Training; System Analysis

\begin{abstract}
The motion simulation is helpful to sports training, which is based on the human method and the change of traditional video acquisition and analysis method of human HD based on analysis method of training methods; from the experience of stylized sports science based on the transfer, thus faster and more effectively to improve the level of sports training and sports performance. Computer simulation of sports in the field of sports has broad application prospects, in recent years has been successfully used in diving, gymnastics, trampoline, weightlifting and other competitive sports. Based on the summary of the motion simulation model, this paper analyzes the characteristics and range of several main modeling methods, and further discusses the visualization technology of motion simulation, and looks forward to its future development trend.
\end{abstract}

\section{Introduction}

Due to the rapid development of computer technology, in order to improve the data storage capacity and the rapid decline in prices, resulting in data storage is a fast and cheap sports players. How to use these massive data, in-depth analysis of technical action essentials, in training to provide athletes and coaches are very useful reference [3]. Therefore, the application of modern computer technology has become one of the important means to improve the level of competition. With many years of research experience, for ordinary sports action, three-dimensional computer processing technology, data model, association rules with regular sports skill characteristic of mining the relationship between the technology of sports action, improve the athletic level, provide the scientific research methods, have a good reference value.

\section{Human joint model overview}

Data can come from human joints motion capture system data (motion capture system) or Microsoft Kinect depth camera obtained after processing. After getting the data key point is how to build a three-dimensional human body model. Human skeleton model is the foundation for the human body model. Because it contains the main points of the joint three-dimensional location information, it can better represent the human information. As used herein human joint model shown in Figure 1, it contains a total of 23 joints, the waist of the root node. Because of the resulting motion capture coordinate data is based on the world coordinate system, we first will coordinate every action will convert to the root of the coordinate origin of the world coordinate system, in order to make the action sequence data regardless of the absolute position of the body [4-5]. 


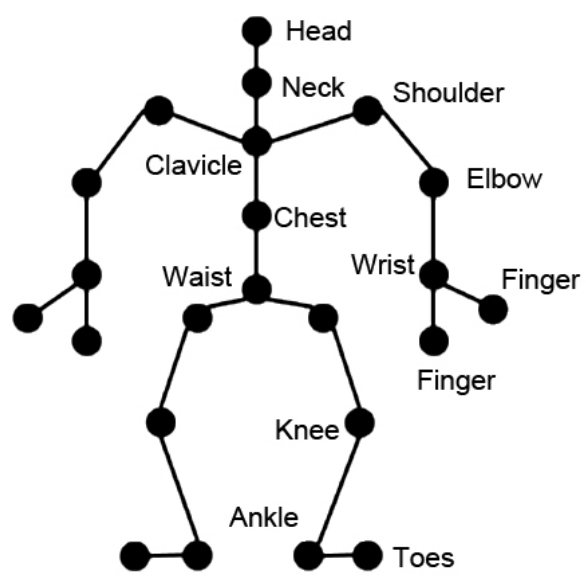

Figure1. 3D human skeleton model

At the same time, the orientation of the body towards the initial orientation is also the $\mathrm{Z}$ direction, so that the initial orientation has nothing to do with human data. At the same time, we will get different people's skeletal length standardized to the same skeleton size, so that the size of the human body movement data independent.

This can be unified as a standard 23 point coordinate joint, the effective data dimension of the 67 dimension (including the height of the root of the cross section information is 0 ). We also break down into a series of human skeletal parts, each of which is a combination of individual joints or joints. Such a node classification contains semantic information, so we extract more efficiently to determine the key part of the operation.

\section{Build sports technical movement features of the model}

Combined with the above analysis and Kinect technique of human joint model design, and in order to construct movement characteristic model conveniently and quickly, the need to classify decomposition and activities on the implementation of the action, game player. The action of database is the key technology to realize the action feature model. As a basic tool of universal unification, the action library should have the characteristics of integrity, independence and generality [6].

In order to realize the integrity and independence of the motion database design, it is necessary to realize the correct classification of the technical action, but in order to achieve the general requirements, we need to ensure the scalability of the library. According to the classification model and movement action task decomposition, we propose a hierarchical structure of the player's library, from top to bottom can be divided into action unit layer, layer, element and technology operation layer, as shown in Figure 2.

Basic action element layer: this layer contains only one basic motilin unit. The basic elements of the action framework define the basic movements of the athletes, and introduce some basic elements, including the start of the operation cycle, pause and stop and the general movement parameters.

The elements of technology action layer: technology elements derived from the basic operation unit of the derived elements, with the basic features of semantic movement, but the movement cannot be divided semantic, is the basic unit of human action. Sports action elements generally follow the human body model and the movement of freedom.

Action unit layer: action unit is composed of a plurality of action elements according to certain rules of semantic, technical operation general semantic features, some semantic features on the description of the activities, but does not include any specific operational semantics related.

The basic layer in action, we limit state based design is a basic action and class action, class action and all elements is the parent class action unit, responsible for implementation of cyclic scheduling techniques; in plane motion element layer, and the basic source package, adjust the 
mentality, hand movement, machine operation dynamic operation unit; motion in the cell layer, further action from the action elements and specific semantic features based on action unit.

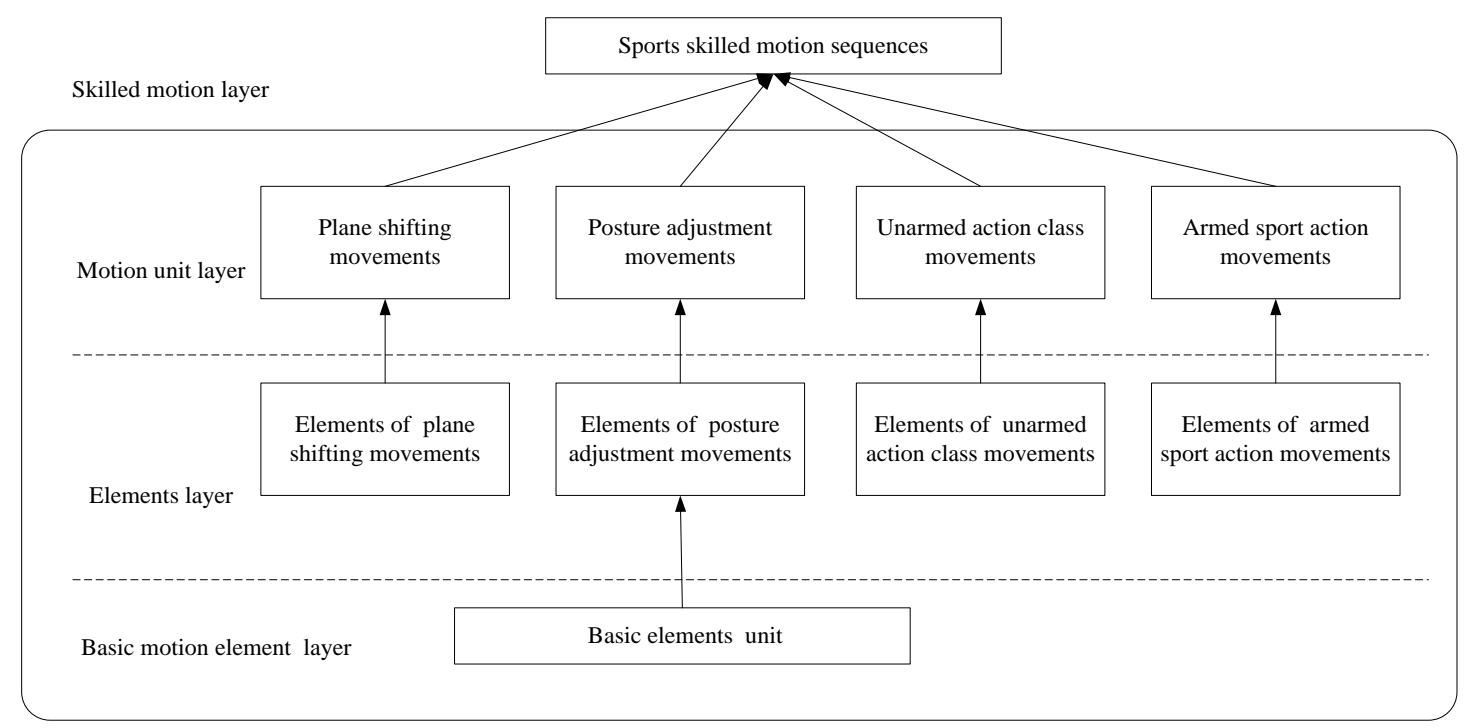

Figure 2.The hierarchical model of sports skilled motion characteristic

\section{D Sport Training Co-Coach Engine achieve}

The realization of 3D game engine includes 3D motion simulation training, analysis, contrast, action design, kinematics analysis, video editing function of six kinds of auxiliary training method in the upgraded version, will join the dynamic analysis. These methods can help athletes understand and master the essentials of physical exercise, help athletes to understand the nature of physical movement from the physical and physical laws, and according to their own circumstances, the development of appropriate training measures. Through the actual situation of virtual players comparison module standard action sports video and motion simulation in the system will display and contrast angle is the same, it can be intuitive, the difference between the two is more accurate, to help athletes improve athletic performance and training efficiency. Analysis of three dimensional motion analysis techniques including kinematics and dynamics analysis. Analysis of the rotation angle of joint movement, analysis of different height, different joint length of athletes and other changes in the same movement in a quantitative way to show the state of the athletes. We choose the focus of joint virtual athletes, in a certain period of time to show the center of gravity trajectory, as shown in Figure 3.

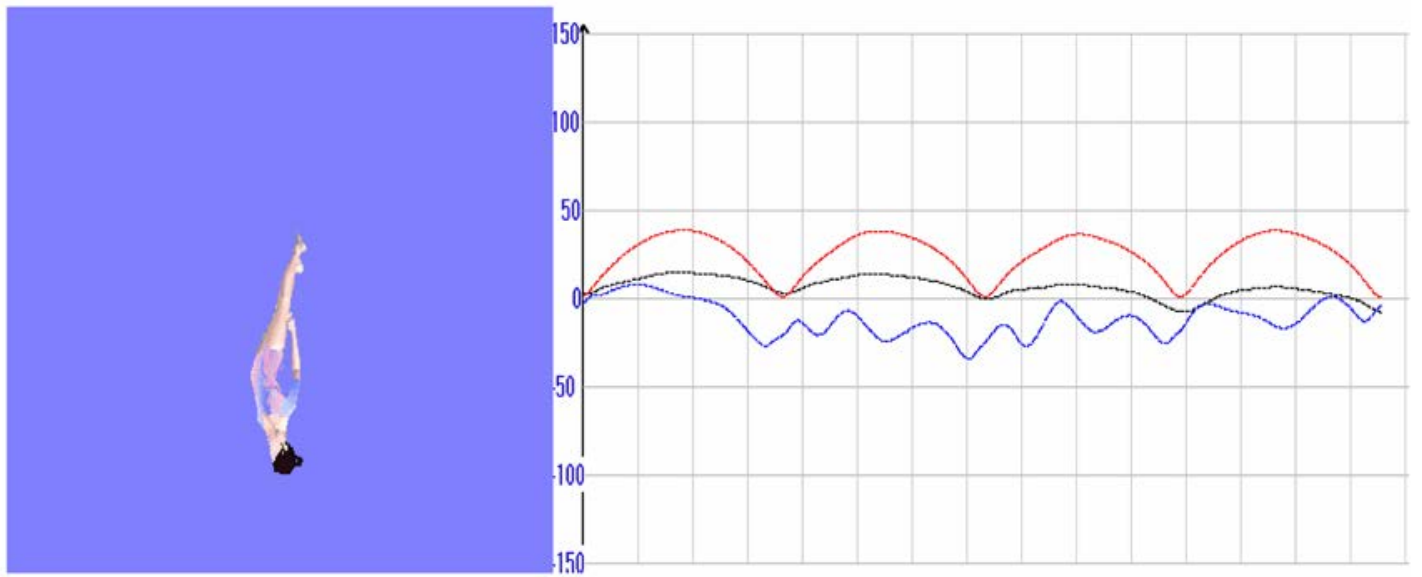

Figure 3. 3D virtual athletes focus locus 


\section{Computer-assisted three-dimensional sports training system analysis}

This system is based on the real-time motion retargeting of human animation fusion: the first automatic extraction of three-dimensional model of three-dimensional skeleton connection graph. Then some skeleton model of the operation of the pretreatment, so that you can complete a skeletal joint CMU matching; automatic driving three-dimensional model of real-time acquisition of Kinect motion data, combined with the redirection technology to ensure the realistic human motion characteristics.

Real-time animation synthesis system designed for dual-threaded, as shown in Figure 4 . The first thread to access real-time capture device motion capture human motion data. The second thread is responsible for loading 3D mesh, 3D mesh models and key point matching skeleton automatic extraction, skin deformation, and motion redirection. 1 thread 2 and thread to communicate and interact through a mutex variable data.

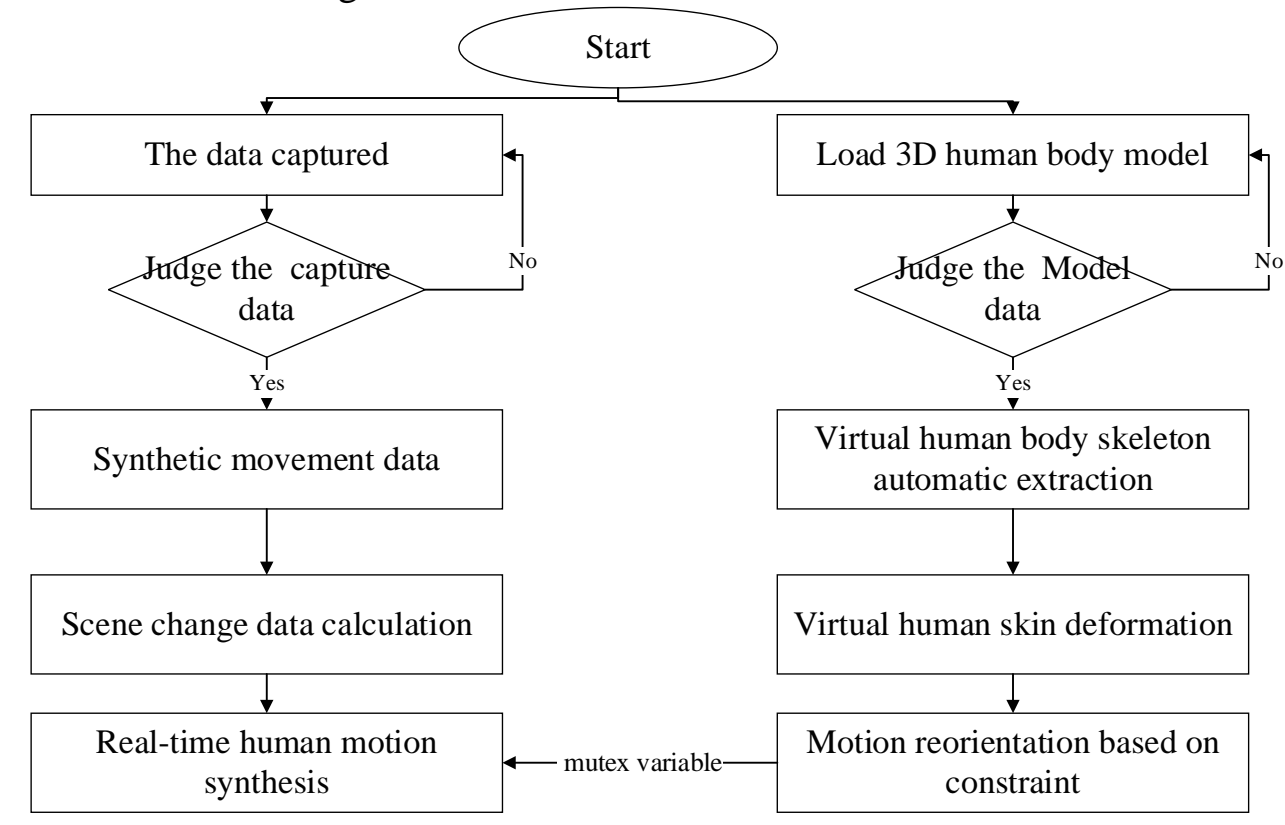

Figure 4. The design flow of Computer-assisted three-dimensional sports training system

The framework includes a number of low visa into high-dimensional data module, three-dimensional grid load, mesh skeleton extraction, matching and real-time motion capture data between nodes driven mesh model. The ultimate goal of the three-dimensional human motion in real-time motion capture system data-driven model, the system modules to achieve other three modules hint. Three-dimensional grid load is a fundamental part of the three modules.

System through the following steps to access the user low-dimensional motion signals into high-dimensional human motion data and accurate:

- According to the position and movement marks a recent synthesis for local sports capture database of choice;

- According to the selected local linear model to generate a motion, and then smoothing model for online motion synthesis;

- According to the root location in the face, the compound new mobile data network, it can be used for animation.

\section{Conclusion}

With the development of 3D computer technology, computer simulation of human motion analysis has been widely used in the field of sports. Computer simulation of human motion key based on human motion model. However, modeling is a difficult and complex task. The traditional method has its own characteristics and advantages, but also has its limitations. Combined with different 
research methods, the use of their complementary advantages will be the focus of research and direction. At the same time, with the development of artificial intelligence technology is introduced, through the simulation of the characteristics of life with the conversion of individual human motion simulation of mechanical properties of the individual campaign staff, who will also be autonomous, intelligent behavior, and the combination and coordination of multiple artificial life behavior, the behavior of workers reflects human's social attribute, this revolutionary change human motion simulation.

\section{Reference}

[1] Ferrari S, Carlomagno N, Di Tore P A, et al. How technologies in the classroom are modifying space and time management in teachers' experience?[J]. REM-RESEARCH ON EDUCATION AND MEDIA, 2013, 5(2): 79-90.

[2] Fogtmann M H, Grønbæk K, Ludvigsen M K. Interaction technology for collective and psychomotor training in sports[C]//Proceedings of the 8th International Conference on Advances in Computer Entertainment Technology. ACM, 2011: 13.

[3] Ochi Y, Takeda Y. Development of a Camera Control System Using Human Gesture Recognition[C]//Hong Kong: Proceedings of the International Multi Conference of Engineers and Computer Scientists. 2013.

[4] Wei Y, Yan H, Bie R, et al. Performance monitoring and evaluation in dance teaching with mobile sensing technology[J]. Personal and Ubiquitous Computing, 2014, 18(8): 1929-1939.

[5] Yamaoka K, Uehara M, Shima T, et al. Feedback of Flying Disc Throw with Kinect and its Evaluation[J]. Procedia Computer Science, 2013, 22: 912-920.

[6] Wattanasoontorn V, Magdics M, Boada I, et al. A kinect-based system for cardiopulmonary resuscitation simulation: a pilot study[M]//Serious Games Development and Applications. Springer Berlin Heidelberg, 2013: 51-63. 